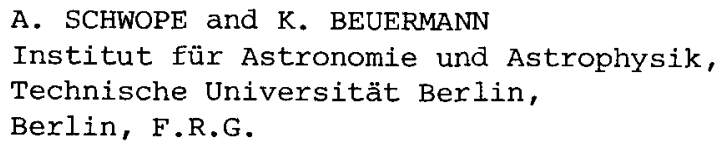

ABSTRACT. We summarize results of our ongoing analysis of optical and $\mathrm{X}$-ray data on the AM Herculis type binary H0139-68 (see also Beuermann et a1., 1984; Schwope and Beuermann, 1985).

\title{
1. EPHEMERIS
}

A reanalysis of published photometric data (Cropper, 1982; Pickles and Visvanathan, 1982; Pickles and Visvanathan, 1983; Thorstensen et al., 1983) together with our $X$-ray and optical observations yields an improved ephemeris

$$
\mathrm{T}_{\mathrm{O}}(\mathrm{HJD})=244 \underset{ \pm 6}{4884.2163}+\underset{ \pm 1}{0.0789152 \mathrm{E},}
$$

which describes the photometric behaviour over a range of about 4 years. $T_{O}$ refers to the start of the bright phase. No systematic difference in the onset times of optical and $\mathrm{X}$-ray bright phases were detected in our observation (Fig. 2).

\section{SECONDARY STAR AND DISTANCE}

The spectrum of the very low state in February $1984(\mathrm{~V}=18)$ is dominated by the stellar components. The spectrum of the red dwarf appears longward of $7000 \AA$ while Zeeman absorption troughs of the magnetized white dwarf strongly affect the continuum between 4000 and $7000 \AA$ (see labels in Fig. 1). The direct measurement of the TiO band strengths turned out to be inaccurate due to the low signal to noise ratio present even in our mean orbital spectrum. The probable occurrence of the vo-band at $7900 \AA$ and the overall flux distribution including IR photometry (Beuermann et al., 1984), however, favour a secondary of very late spectral type, M6-M7. The value of the colour $\mathrm{H}-\mathrm{K}=0.61 \pm 0.13$, measured when the system was in a similar or even lower state, is larger than that of known late $M$-dwarfs (Probst and

Paper presented at the IAU Colloquium No. 93 on 'Cataclysmic Variables. Recent Multi-Frequency Observations and Theoretical Developments', held at Dr. Remeis-Sternwarte Bamberg, F.R.G., 16-19 June, 1986. 
Liebert, 1983), possibly indicating the presence of still another radiation component in the infrared. The $M$-dwarf G51-15 seems to qualify as a template for H0139-68. The absolute K-magnitude of G51-15 then yields a distance modulus of $4.7 \pm 0.3$ and a distance of about 90 pc. Bailey's (1981) method yields a similar result, if we use a slightly different $S_{K}$ vs. ( $\left.V-K\right)$ relation appropriate for the latest $M-$ stars, $\mathrm{S}_{\mathrm{K}}=5 \cdot 0-5.5$ for $\mathrm{V}-\mathrm{K}>6$. Given the distance, the temperature of the white dwarf can be estimated to be $T_{\text {eff }} \leqslant 20000 \mathrm{~K}$ (Wesemael et a1., 1980), assuming a standard white dwarf.

\section{LOW-STATE LIGHT CURVES AND CYCLOTRON SPECTRUM}

The shape of the optical light curve is subject to minor changes only as the system switches from the high state (Pickles and Vivanathan, 1983; Thorstensen et al., 1983) to the low state (1984 Oct 23, V = 16.9-17.3). The $X$-ray light curve in the low state again resembles the optical light curve and both results taken together are suggestive of a one-pole geometry. The accreting pole is occulted by the white dwarf for about $50 \%$ of the orbital period. The optically bright phase seems to be dominated by cyclotron radiation. On this assumption, we derived a mean low-state cyclotron spectrum by subtracting mean bright and

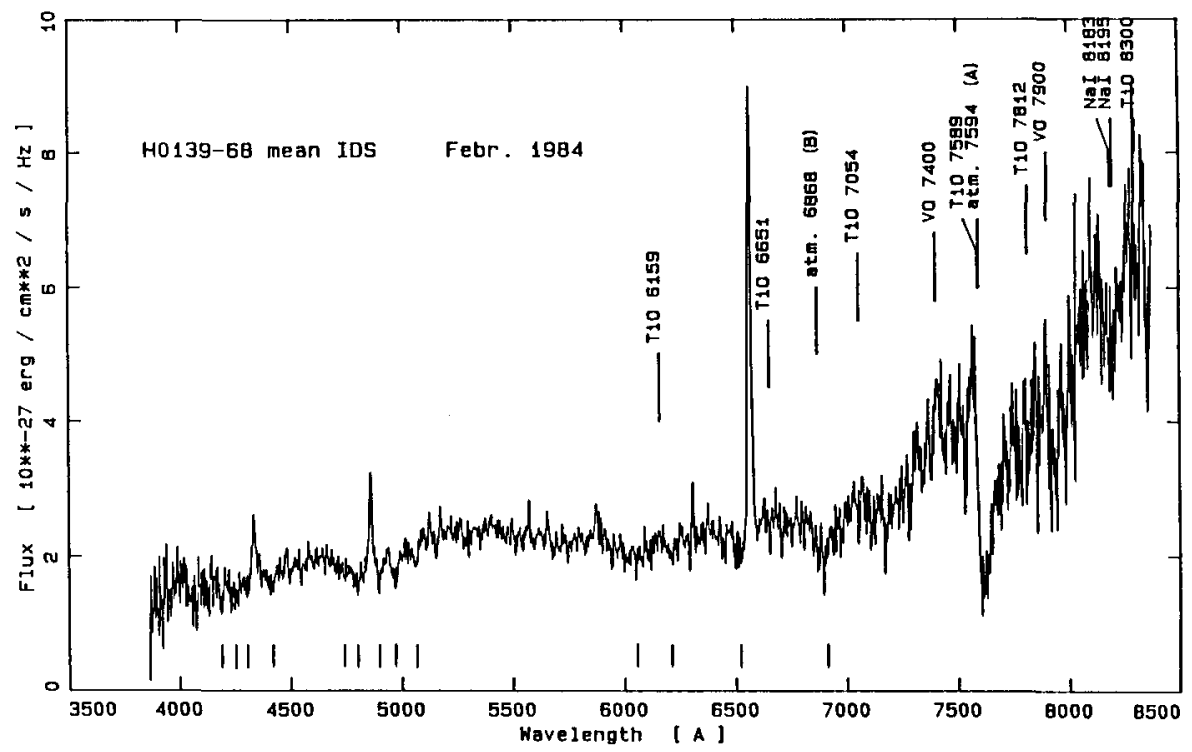

Figure 1. Spectrophotometry of H0139-68 in February 1984. Typical Mstar features are labeled. Vertical marks below the spectrum indicate the positions of zeeman absorption troughs. The flux calibration may be inaccurate for $\lambda \lesssim 5000 \AA$ because of light loss due to differential refraction. 
faint phase spectra (Fig. 3). The same procedure applied to the highstate data of Visvanathan and Pickles (1982) yields the cyclotron spectrum for the high state (Oct. 81). For our favorite geometry, $i \simeq$ $25^{\circ}$ and $\delta \simeq 98^{\circ}$ (Schwope and Beuermann, 1985), both spectra correspond to a mean angle between the line of sight and the magnetic axis of $\theta \simeq$ $80^{\circ}$. Comparison with the theoretical cyclotron spectra of Wickramasinghe and Meggitt (1985) yields best agreement for temperatures $\mathrm{kT}=10-20 \mathrm{keV}$ and size parameters $\Lambda=10^{4}-10^{5}$. Highstate and low-state fluxes differ by about a factor of 30 . Because the temperatures seem to be similar, we may expect a corresponding difference in the emitting areas between the two states. The hard bremstrahlung source is clearly present in the low state but again weaker than observed in the high state (Agrawal et al., 1983), suggesting that also the bremstrahlung-emitting area was significantly reduced in the low state. In addition, a soft component with temperature $\mathrm{kT}$ in the range of $10-30 \mathrm{eV}$ is present.

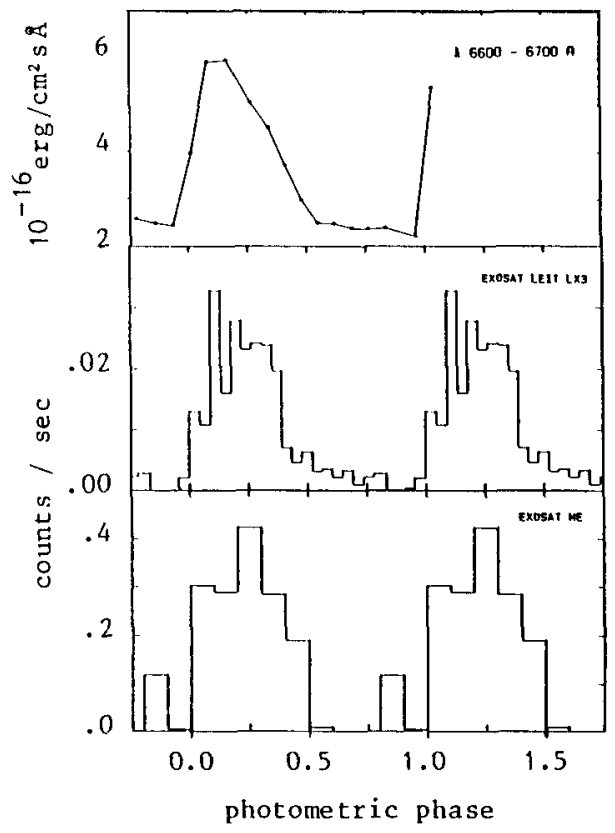

Figure 2. Simultaneous X-ray/optical observations of H0139-68 on 1984, Oct. 23. Shown are (from top to bottom) the variation of the continutm flux between 6600 and $6700 \AA$ and the EXOSAT light curves obtained with the low-energy telescope with CMA and $3000 \AA$ Lexan filter $(0.05-2 \mathrm{keV})$ and with the medium-energy detector $(1-10 \mathrm{keV})$. The X-ray light curves are corrected for background and folded over the orbital period using the ephemeris given above. The optical light curve is not folded; the start time is $02^{\mathrm{h}_{01}} \mathrm{l}^{\mathrm{m}}$ HUT; integration times of the individual CCD frames are 7 minutes. 


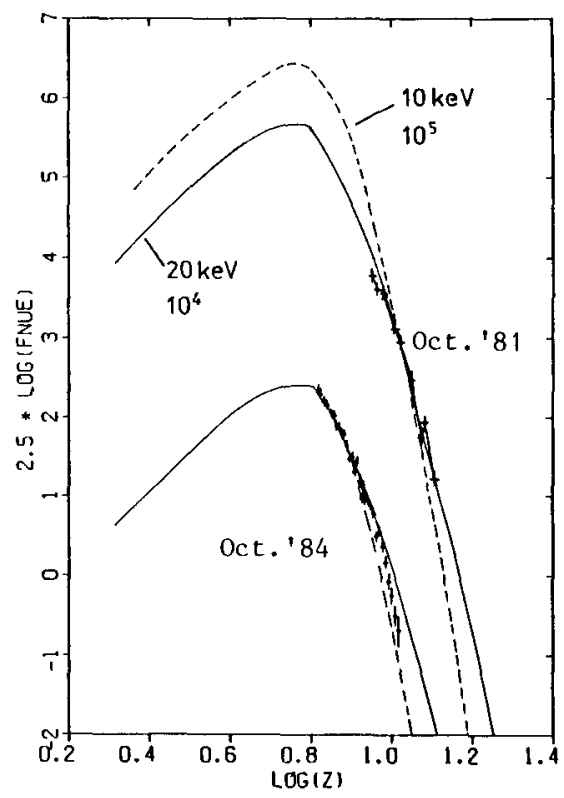

Figure 3. Cyclotron spectra of H0139-68 in the high and the low state. A mean magnetic field strength of 20 MG was used to scale the frequency axis. $Z$ indicates the harmonic number. The model spectra refer to temperatures of 10 or $20 \mathrm{keV}$, size parameter $\Lambda$ of $10^{4}$ or $10^{5}$ and $\theta=80^{\circ}$ (Wickramasinghe and Meggitt, 1985). Flux units are $\left.10^{-2}\right\}$ $\mathrm{erg} / \mathrm{cm}^{2}$ s $\mathrm{Hz}$.

\section{References:}

Agrawa1, P.C., Riegler, G. R., and Rao, A. R., 1983, Nature 301, 318. Bailey, J., 1981, Mon. Not. R. astr. Soc. 197, 31 .

Beuermann, K., Schwope, A., Weißsieker, H., and Motch, C., 1984, Space Sci. Rev. 40,135 .

Cropper, M. S., 1982, Inf. Bull. Var. Stars No. 2096.

Pickles, A. J., and Visvanathan, N., 1982, Proc. ASA, 4(4), 425.

Pickles, A. J., and Visvanathan, N., 1983, Mon. Not. R. astr.Soc. 204, 463.

Probst, R. G., and Liebert, J., 1983, Astrophys. J. 274, 245.

Schwope, A., and Beuermann, K., 1985, In: W. R. Burke (Editor), Proc. ESA Workshop: Recent Results on Cataclysmic Variables, Bamberg, ESA SP-236, p. 173 .

Thorstensen J. R., Schommer, R. A., and Charles, P. A., 1983, PASP 95, 140.

Visvanathan, N., and Pickles, A.J., 1982, Nature 298, 41.

Wesemael, F., Auer, L. H., von Horn, H. M. , and Savedoff, M. P., 1980, Astrophys. J. Supp1. 43, 159.

Wickramasinghe, D. T., and Meggit, S. M. A., 1985, Mon. Not. R. Soc. 214, 605 . 